\title{
Double Trouble: A Rare Association between Chronic Total Occlusion of the Left Main Coronary Artery and Colonic Malignancy
}

\author{
Krishna Prabu Ramaraj ${ }^{4}$ Anto Mariadoss Fernando ${ }^{5}$ \\ 1Department of Cardiology, Sri Manakula Vinayagar Medical College \\ and Hospital, Pondicherry, India \\ ${ }^{2}$ Cardiothoracic and Vascular Surgery, Sri Manakula Vinayagar \\ Medical College and Hospital, Pondicherry, India \\ ${ }^{3}$ Department of General Medicine, Sri Manakula Vinayagar Medical \\ College and Hospital, Pondicherry, India \\ ${ }^{4}$ Department of Anaesthesiology, Sri Manakula Vinayagar Medical \\ College and Hospital, Pondicherry, India \\ ${ }^{5}$ Department of General Surgery, Sri Manakula Vinayagar Medical \\ College and Hospital, Pondicherry, India
}

Ashida Thulaseedharan Sarojadevi ${ }^{1}$ Arunkumar Arasappa ${ }^{2}$ Priscilla Rubavathy Eugin ${ }^{3}$

\begin{abstract}
Address for correspondence Priscilla Rubavathy Eugin, MBBS, MD, No. 5, 2nd Cross Street, Kumaran Nagar, Lawspet, Pondicherry 605008 , India (e-mail: priscilla.rubavathy@gmail.com).
\end{abstract}

J Health Allied Sci Nu:2021;11:47-49

\begin{abstract}
Emerging evidence has shown an association between cardiovascular (CV) disease and cancer due to shared risk factors and biological mechanisms especially chronic inflammation. The objective of this case report is to highlight the association between these two lethal diseases and the challenges in the management of coronary artery disease in patients with coexisting malignancy.

A 65-year-old nonsmoker, nondiabetic, and normotensive male presented with a history of abdominal pain and significant weight loss. Colonoscopy and biopsy showed adenocarcinoma of the ascending colon, and he was planned for right hemicolectomy. Electrocardiogram exercise stress test performed as a part of preoperative evaluation was strongly positive. Coronary angiography was suggestive of Chronic total occlusion of the left main coronary artery. Though the syntax score was intermediate, coronary artery bypass grafting was decided as the revascularization strategy as he needed early surgery for the colonic malignancy. A month later, he underwent right hemicolectomy.

Keywords

- chronic total occlusion

- LMCA

- colonic malignancy

Clinicians should be aware of the association between CV disease and cancer as they are likely to face similar situations where both coexist. Understanding the connections between heart disease and cancer will help to formulate combined preventive guidelines.
\end{abstract}

\section{Introduction}

Chronic total occlusion (CTO) of the left main coronary artery (LMCA) is uncommon, and survival depends on good collaterals from the right coronary artery (RCA). Emerging evidence suggests a relationship between cancer and coronary artery disease (CAD) due to shared risk factors and biological mechanisms especially chronic inflammation.

The management of CAD in cancer patients can be challenging due to issues related to cancer treatment or the published online

November 10, 2020
DOI https://doi.org/

$10.1055 / \mathrm{s}-0040-1721154$

ISSN 2582-4287.

\footnotetext{
(c) 2020. Nitte (Deemed to be University).

This is an open access article published by Thieme under the terms of the Creative Commons Attribution-NonDerivative-NonCommercial-License, permitting copying and reproduction so long as the original work is given appropriate credit. Contents may not be used for commercial purposes, or adapted, remixed, transformed or built upon. (https://creativecommons.org/licenses/by-nc-nd/4.0/)

Thieme Medical and Scientific Publishers Pvt. Ltd., A-12, 2nd Floor, Sector 2, Noida-201301 UP, India
} 
cancer itself. Here we report a case of a patient who had CTO of LMCA with coexisting colonic malignancy.

\section{Case Presentation}

A 65-year-old male presented to the general surgery outpatient department with complaints of abdominal pain for 4 months aggravated for the last 2 months. He had loss of appetite and significant weight loss. His body mass index was $19 \mathrm{~kg} / \mathrm{m}^{2}$ (height: $154 \mathrm{~cm}$; weight: $45 \mathrm{~kg}$ ). There was no history of vomiting or bleeding per rectum. He was a nonsmoker, nonhypertensive, and nondiabetic. General physical examination showed significant pallor.

Laboratory investigations were normal except for low hemoglobin levels. Colonoscopy was performed, which showed a growth in the ascending colon. Biopsy report showed adenocarcinoma of the ascending colon, and the patient was planned for right hemicolectomy.

A routine preoperative cardiac evaluation was performed. Electrocardiogram (ECG) was suggestive of lateral wall ischemia. Two-dimensional echocardiogram showed normal left ventricular (LV) function with no regional wall motion abnormalities. On repeated questioning, the patient reported only NYHA (New York Heart Association) class 2 dyspnea, which was present for more than 2 years but did not interfere with his daily activities. ECG exercise stress test was performed on July 3, 2019, which was strongly positive (-Fig. 1).

Coronary angiography was performed through the transradial route after informed patient consent.

The LMCA could not be engaged with a 6-Fr Tiger catheter. Nonselective injection in the left sinus did not reveal the LMCA. RCA angiogram showed filling of the LMCA, left anterior descending (LAD), and the left circumflex branches by Rentrop grade 3 collaterals. There was no significant RCA stenosis. The coronary angiography was suggestive of CTO of LMCA ( - Fig. 2).
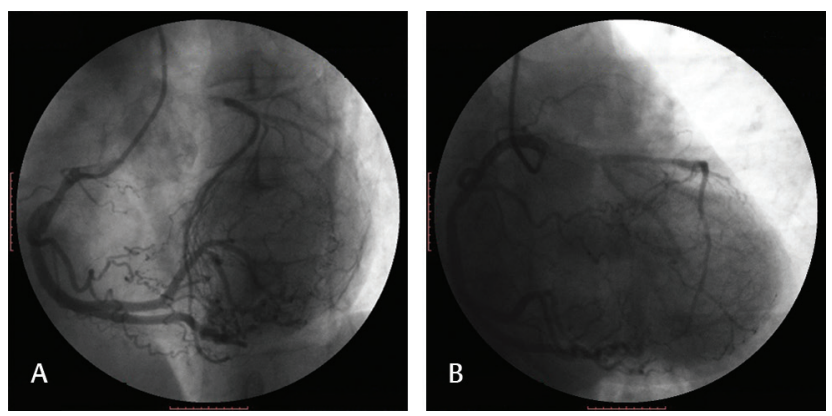

Fig. 2 (A,B) Right coronary angiogram showing retrograde filling of the left coronary system by collaterals from the dominant right coronary artery.

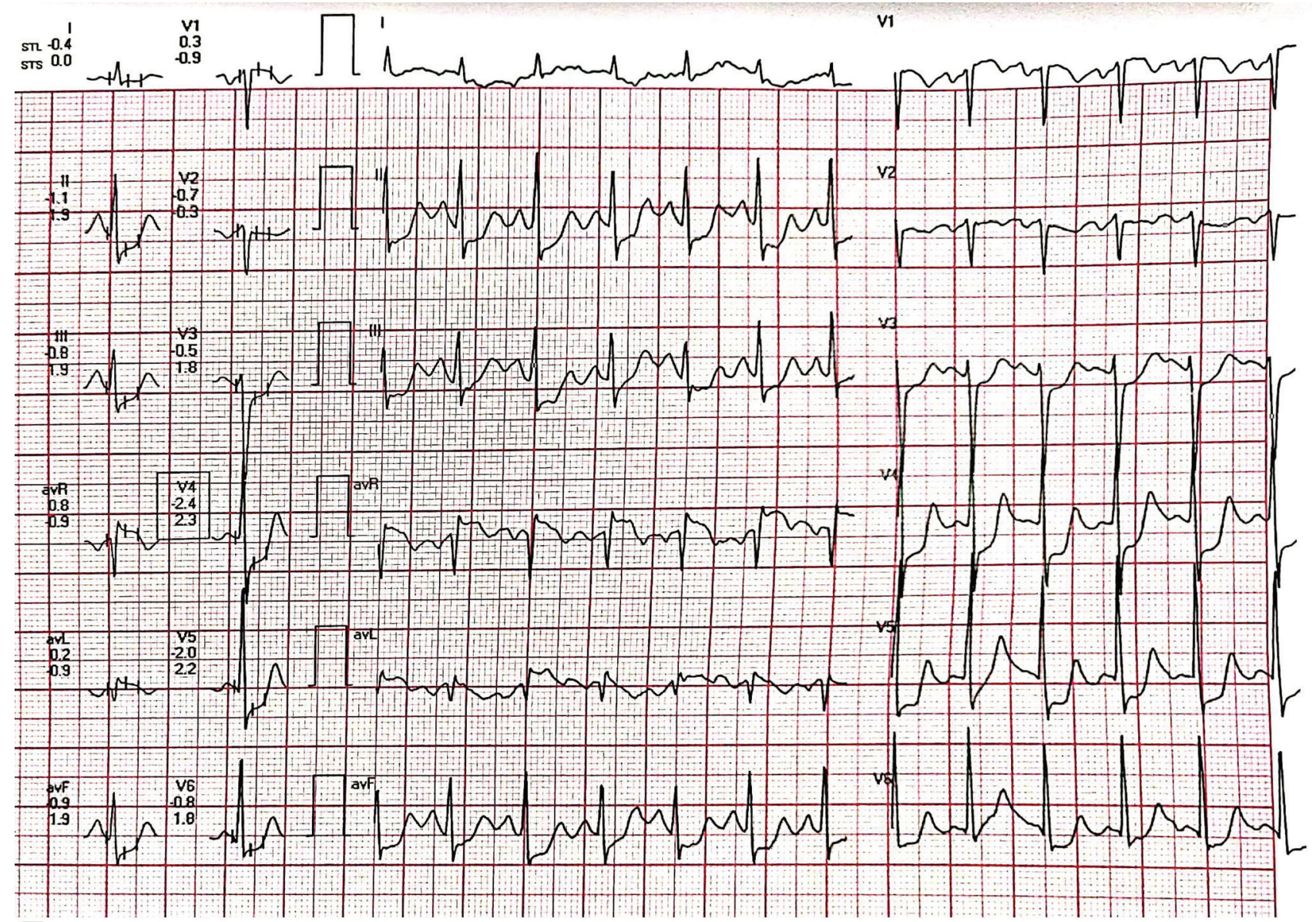

Fig. 1 Treadmill test tracing showing 2-mm ST depression in more than five leads. 
Treatment options were discussed by a team including a cardiologist, cardiothoracic surgeon, oncologist, and surgeon. Coronary artery bypass grafting (CABG) was planned for the LMCA occlusion as the patient needed early abdominal surgery. The patient underwent CABG with grafts to LAD and obtuse marginal on July 5, 2019. He was started on double antiplatelets (clopidogrel and aspirin).

A month later, he underwent right hemicolectomy. The dual antiplatelets were withheld 3 days before the procedure and restarted in the perioperative period. At present, the patient is under regular follow-up and is on antiplatelets and statins.

\section{Discussion}

This case highlights a rare association between CTO of LMCA and right colon cancer, where the patient was asymptomatic for the CAD.

CTO of LMCA is uncommon, and the prevalence varies from 0.04 to $0.4 \% .{ }^{1}$ Patients with this critical lesion can have clinical presentations varying from stable angina, heart failure, to acute coronary syndromes. Symptoms, survival, and maintenance of LV function depend on dominance of RCA, extent of collateral network from the right to left coronary system, and the absence of right coronary lesion. ${ }^{2}$

Our patient reported no previous angina and total occlusion of the LMCA was diagnosed at his index presentation for colonic malignancy. This was due to the absence of significant disease in a dominant RCA and the presence of good collateral connections.

Community-based observational trials have suggested a relationship between CAD and cancer. ${ }^{3}$ Both diseases can occur in the same patients due to shared risk factors such as obesity, tobacco use, hyperglycemia, sedentary lifestyle, and dietary factors. Chronic inflammation is the major unifying biological mechanism linking the two diseases. ${ }^{4}$ Studies have reported an association between colorectal neoplasia and CAD, especially right-sided colon cancer (RCC). ${ }^{5,6}$ This patient, who did not have any major cardiovascular (CV) risk factors, was found to have a coassociation between RCC and CTO of LMCA.

The management of CAD in patients with coexisting malignancy is challenging due to issues related to both treatment of the malignancy and the malignancy per se. Recent randomized trials have shown that percutaneous coronary intervention for LMCA stenosis is a safe option and has similar long-term survival rates to CABG surgery in select patients with less complex anatomy (SYNTAX score of <33). ${ }^{7,8}$ In our patient, though the syntax score was intermediate, CABG was decided as the revascularization strategy as he needed early surgery for the colonic malignancy. This again highlights the impact of one disease over the management of the other.

Current studies suggest an increased incidence of colorectal cancers in India as well as all over the world, especially in the younger age group. ${ }^{9}$ Since the incidence of CAD is also on the increase, clinicians are likely to face similar situations where CAD and malignancies coexist. Cardiac risk factors also influence toxicity from cancer therapy. Studies have demonstrated that controlling $\mathrm{CV}$ risk factors can reduce the risk of incident cancer over time. ${ }^{10}$

\section{Conclusion}

Many risk factors are shared between CV disease and cancer, resulting in a strong association between these two lethal diseases. Both diseases may coexist in the same patient, or the diagnosis of one may precede the other. Clinicians should be aware of this overlap as significant CAD may be relatively asymptomatic in patients with cancer, as in our case. CV risk factors have an impact on cancer treatment related cardiotoxicity. Understanding the connections between heart disease and cancer will help formulate combined preventive guidelines.

\section{Conflict of Interest}

None declared.

\section{References}

1 Zimmern SH, Rogers WJ, Bream PR, et al. Total occlusion of the left main coronary artery: the Coronary Artery Surgery Study (CASS) experience. Am J Cardiol 1982;49(8):2003-2010

2 Valle M, Virtanen K, Hekali P, Frick MH. Survival with total occlusion of the left main coronary artery. Significance of the collateral circulation. Cathet Cardiovasc Diagn 1979;5(3):269-275

3 Hasin T, Iakobishvili Z, Weisz G. Associated risk of malignancy in patients with cardiovascular disease: evidence and possible mechanism. Am J Med 2017;130(7):780-785

4 Koene RJ, Prizment AE, Blaes A, Konety SH. Shared risk factors in cardiovascular disease and cancer. Circulation 2016; 133(11):1104-1114

5 Wang SC, Schulman-Marcus J, Fantauzzi J, et al. Colon cancer laterality is associated with atherosclerosis and coronary artery disease. J Gastrointest Oncol 2019;10(1):30-36

6 Niederseer D, Stadlmayr A, Huber-Schönauer U, et al. Cardiovascular risk and known coronary artery disease are associated with colorectal adenoma and advanced neoplasia. J Am Coll Cardiol 2017;69(18):2348-2350

7 Morice MC, Serruys PW, Kappetein AP, et al. Five-year outcomes in patients with left main disease treated with either percutaneous coronary intervention or coronary artery bypass grafting in the synergy between percutaneous coronary intervention with taxus and cardiac surgery trial. Circulation 2014; 129(23):2388-2394

8 Stone GW, Sabik JF, Serruys PW, et al; EXCEL Trial Investigators. Everolimus-eluting stents or bypass surgery for left main coronary artery disease. N Engl J Med 2016;375(23):2223-2235

9 Pal M. Proportionate increase in incidence of colorectal cancer at an age below 40 years: an observation. J Cancer Res Ther 2006;2(3):97-99

10 Rasmussen-Torvik LJ, Shay CM, Abramson JG, et al. Ideal cardiovascular health is inversely associated with incident cancer: the Atherosclerosis Risk In Communities study. Circulation 2013; 127(12):1270-1275 